\title{
Numerical simulation of the sliding wear test in relation to material properties
}

\author{
N. Stalin-Muller ${ }^{a}$, K. Dang Van ${ }^{b}$ \\ - Laboratoire de Mécanique de l'Ecole Nationale Supérieure de Techniques Avancées, Centre de l'Yvette, Chemin de la Hunière, 91120 Palaiseau. France \\ 'Laboratoire de Mécanique des Solides de l'Ecole Polytechnique. 91128 Palaiseau Cedex, France
}

\begin{abstract}
Although many wear criteria have been proposed, none is intrinsic to the materials and to the structures in contact. We propose new computational methods for modelling the wear phenomenon and to improve the meaning of these criteria. To interpret sliding wear experiments, numerical calculations have been performed to estimate the mechanical state in the vicinity of the wear region and to infer the dissipation mechanism activated. Calculations in two and three dimensions are proposed to estimate the stress, and the total and plastic strain cycles near the contact under repeated loadings. A result is the stress and strain amplitudes which are derived for different hertzian loadings based on measured friction coefficients. To evaluate wear and fatigue properties, plastic strain amplitude and dissipation rate are also estimated. The modification induced by wear of the original contact geometry is accounted for following the proposals of Kapoor and Johnson (J. Mech. Phys., Solids, 34 (3) (1992) 223-239). The initial contact geometry (circular) is modified by wear so that the contact surface increases and the contact stress decreases. To account for these effects, computations are made with different geometries and hertzian pressure varying from initial conditions to the stabilized state corresponding to shakedown conditions. The plastic strain amplitude and the rachetting rate evolution are then obtained.
\end{abstract}

Keywords: Numerical simulation: Sliding wear test: Material properties

\section{Introduction}

A diversity of wear models have been proposed in the past but few can really be ised by engineers and designers. In a recent paper Meng and Ludema [1] analyse the main reasons and conclude that "It is unlikeiy that many applicable equations will emerge using the approach of the past" in spite of the great number of research works already done. One of the main difficulties in modelling comes from the complexity of evaluating the thermo-mechanical state (or even the chemico-thermo-mechanical state) in the vicinity of regions submitted to wear. From a fundamental point of view, several mechanisms can be at the origin of wear, such as, for example, cutting or plastic burnishing in a ploughing process between asperities in abrasive wear. Various authors have proposed that wear takes place by a fatigue process and that the $\boldsymbol{K}$ coefficient of Archard's wear law is a measure of the number of the load cycle (or repeated asperity encounters) to produce rupture. However, deriving wear laws from fatigue theories is not an easy task for two principal reasons. First, it is a multiaxial fatigue phenomenon for which very few criteria can be applied successfully. Second, one has to evaluate the multiaxial stress and strain tensors in the regions where wear occurs, which was not really done until now.

Recently Kim and Ludema [2] have given an interesting correlation between scuffing and low cycle fatigue properties of $\mathbf{4 3 4 0}$ steel. However they were not able to evaluate the mechanical varicibles such as stress or strain amplitude which enter the fatigue laws. Consequently they could not continue their quantitative analysis. Recently, Johnson [3] published an important contribution entitled "Contact mechanics and the wear of metals" in which he recalled the main analysis necessary to estimate the mechanical state in the wear region. His reasoning is based on an interpretation of the wear mechanism as a fatigue or ductile fracture process. This is suggested by experimental obseryations using scanning electron microscope and ferrog:aphy. Johnson concludes that "I now take the view that delamination wear cracks are indeed shear cracks, but of a ductile nature, driven by plastic strain rather than elastic stress intensity". In this process rachetting is an important phenomenon because it induces large accumulation of plastic deformation which can lead to the detachment of particles. 
Various approaches have been followed up to now to estimate the mechanical parameters in the wear region. Three of these deserve some discussion.

- The slip line field method for plane strain deformation of rigid, perfectly plastic materials of Challen and Oxley [4] provides the rachetting strain resulting from each cycle. However, this method overestimates the rachetting effect because it disregards the elastic strains.

- Shake-down theorems have been applied to provide the elastic and plastic shake-down limits (shake-down map) for a hard cylinder rolling and/or sliding on an elastic plastic half-space.

- The approximate methods of Bower and Johnson [5] provide an estimate of the ratchet rate for specific values of the friction coefficient.

Another procedure to estimate the mechanical state near the contact is to consider an elasto-plastic model and a finite element method. This was done, for instance, by Barghava et al. [6] with an incremental translation of the loading. Such an approach is rather involved and the authors are limited to a small number of cycles, which is insufficient to provide a precise idea of the stabilized ratchetting rate.

More recently, Dang Van and Maitournam developed a systematic and reliable finite element algorithm for thermoelasto-plastic solids which is well adapted for repeated roll:..1g and sliding contact problems [7-9]. Their method also avoids lengthy and repeated calculation resulting from an incremental analysis of the loading history. This method is based on a steady state assumption for an observer moving with the contact load. Two calculations procedures were proposed. The first is the pass by pass stationary method (PPSM) and the second the direct stationary method (DSM) for a repeated moving load. This second provides directly the stabilized state. Recently these methods have been extended to three-dimensional cases by Maitournam et al. [10].

The purpose of this paper is to present applications of these new computational methods to wear problems. Our objective is to derive stress, plastic strain tensors and their variations, in particular the ratchetting rate, in contact regions where wear may happen in relation with the loading conditions and the local friction coefficient $\mu$. This work constitutes a first step for a quantitative understanding of wear phenomena.

\section{The numerical method}

The steady-state assumption is proposed for an observer moving with the loading. This means that, in this reference frame, all physical and mechanical quantities are time independent and only position dependent along the moving direction. Furthermore, the hypothesis of small strains, permits derivation of the evolution equations governing our problem. Disregarding the thermomechanical couplings, these equations are, the equation of motion or of equilibrium, the boundary and initial conditions and the constitutive equations.



Disk : $D=40 \mathrm{~mm}, \mathrm{~h}=8 \mathrm{~mm}$ Sphere : $\$=6 \mathrm{~mm}$.

$\theta$ is angular position of the almans epphare aliating on the wear path.

Fig. 1. Description of the experimental set-up rodelled numerically in the space $(r, \theta, z)$.

In practice, the main difference between our method and a classical integration scheme lies in the determination of the plastic strain rate and the related internal hardening parameters. For instance, for a pin pressed against a rotating disk (Fig. 1), one has

$\frac{d}{d t}=-\omega \frac{d}{d \theta}$

in which $\omega$ is the angular velocity, so that the usual plastic strain rate

$\dot{\boldsymbol{\epsilon}}_{\mathrm{p}}=\lambda \frac{\partial f}{\partial \sigma}$

should be replaced by

$\frac{\partial e^{p}}{\partial \theta}=-\lambda^{*} \frac{\partial f}{\partial \sigma}$

where $\lambda$ is the plastic multiplier and $f$ is the yield function.

The plastic strain tensor at any point (defined by its coordinates $r, \theta$ and z) can be evaluated by integration along the loading path:

$\left[\epsilon^{p}(r, \theta, z)\right]_{\theta}^{2 \pi}=-\int_{\theta}^{2 \pi} \lambda^{*} \frac{\partial f}{\partial \sigma}$

$\epsilon^{P}(r, \theta, z)=\int_{\theta}^{2 \pi}\left(\dot{\lambda}^{*} \frac{\partial f}{\partial \sigma}\right)(r, \xi, z) d \xi+\epsilon^{P}(r .2 \pi, z)$

Fourier's series are convenient for these three-dimensional problems in view of the contact geometry. This method, proposed by Zienkiewicz, was adapted for the cyclic elastoplastic problem by Maitournam et al. [ 10].

\section{Description of the studied system}

Our numerical simulation concerns the classical experiment set-up used by, for example, Papaphilippov [11] on the 


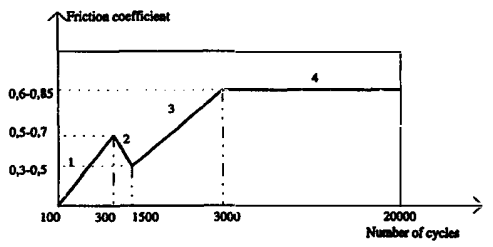

Fig. 2. Evolution in time of the friction coefficient during wear tests on a cast iron disk [11].

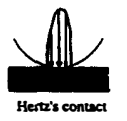

3.

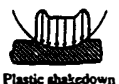

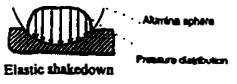

$3 c$

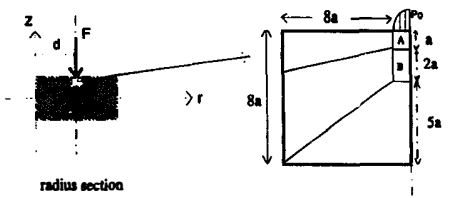

Fig. 4. Mesh in the section of the sliding path.

wear phenomena induced by an alumina sphere sliding on a rotating cast iron disk. The experimental set-up is shown in Fig. 1.

The friction coefficient is found experimentally to increase during the wear test as shown in Fig. 2. This variation is explained by the formation of small oxide islands ( 1 in Fig. 2), a generalization of oxidation (2), and the removing of the oxide film: (3) prior to the stabilization (4). We disregard the transition phase during the numerical simulation. The local friction coefficient is thus taken as constant.
Table i

Material properties of cast iron



During the test, the hard alumina sphere remains elastic but the cast-iron disk undergoes plastic deformation. This deformation increases the conformity between the two surfaces, thereby reducing the contact stress and promoting shakedown. The evolution of the contact characteristics is schematized in Fig. 3(a), 3(b) and 3(c) which represents the evolution in three steps of the contact area pressure distribution during the wear test.

The mesh used for the simulation concerns the section of the wear path which belongs to the $(r, z)$ plane. The surface of contact being very small, the mesh situated near the loading is very fine. In particular, the mesh size near the contact is approximately one tenth of $a$. Fig. 4 gives an idea of the discretization. The two zones $A$ and $B$ are finely meshed (up to 300 elements).

The cast iron is assumed to obey a linear kinematic hardening law. Its mechanical characteristics are listed in Table 1.

\section{Numerical simulations}

Three levels of loading are considered: $10 \mathrm{~N}, 15 \mathrm{~N}$ and 20 N. Contact parameters (the radius $a$ of the initial contact zone, $\boldsymbol{P}_{\mathrm{o}} / \boldsymbol{k}$ which is the maximum normalized pressure) are first evaluated with Hertz's elasticity theory. The results obtained are presented in Table 2.

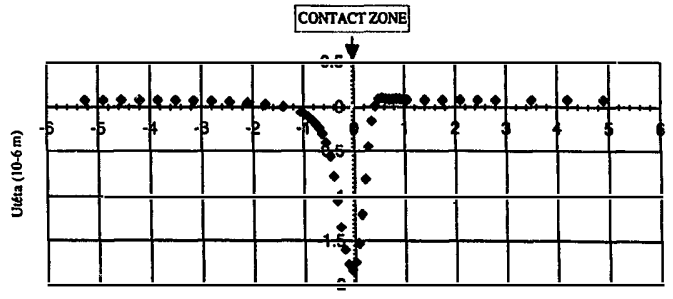

ANGUI_AR POSTIION TETA (rd)

Fig. S. Distribution of the tangential displacement $U_{0}$ versus $\theta$ (rad), $F=10 \mathrm{~N}, \mu=0.5$. 
$P_{\mathrm{o}} / k$ exceeds the elastic limit which is approximately equal to 3 as shown by Johnson [12]. Consequently the real radius should be greater and the maximum $\boldsymbol{P}_{\mathrm{o}}$ lower than the values in Table 1, so that the pressure distribution should differ from that predicted by Hertz's theory. Nevertheless, this proposed distribution is an approximation for our initial computations.
For repeated loadings the distribution should tend towards the hertzian distribution even if the imposed load exceads the static elastic limit providing that $\boldsymbol{P}_{\mathrm{o}} / \boldsymbol{k}$ is below the elastic shakedown limit.

As an example, let us consider the following case corresponding to a $10 \mathrm{~N}$ load and a friction coefficient $\mu$ equal to

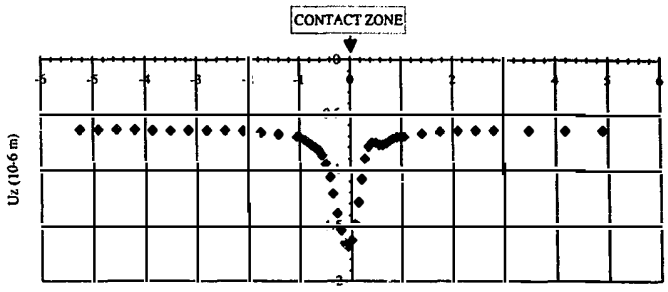

ANGULAR POSITON TETA (rd)

Fig. 6. Distribution of the nornal displacement $U_{2}$ versus $\theta(\mathrm{rad}), F=10 \mathrm{~N}, \mu=0.5$.



ANGUUARFOSTION TETA (rO)

Fig. 7. Distributionof the plastic shear strain $\epsilon_{2}^{\text {Z versus }} \theta$ (rad), $F=10 \mathrm{~N}, \mu=0.5$ (plastic shakelowo stare).

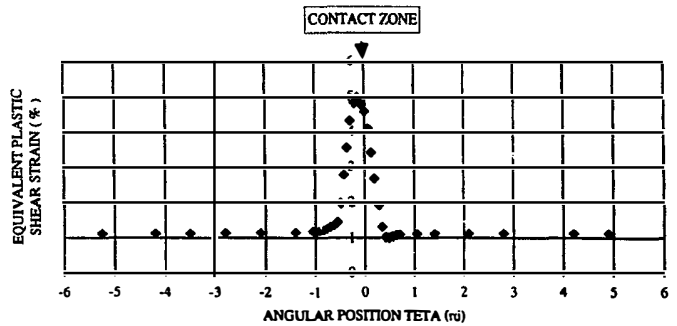

Fig. 8. The maximal plastic shear suain 


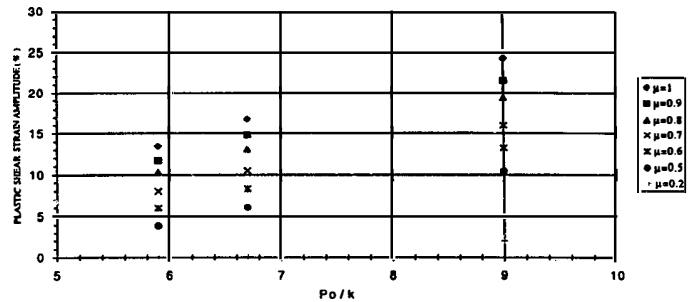

Fig. 9. The plastic shear strain amplitude $\Delta \epsilon_{\frac{Q}{2}}^{p}$ for the three levels of loading.



\section{$\mu=1$}

$\boldsymbol{D}=0.9$

$\Delta \mu=0.82$

$\times \mu=0.7$

$X \mu=0.6$

$0=0.5$

$+\mu=0.2$

Fig. 10. Dissipation per cycle rate, $E_{0}$ for three levels of loadings.

Table 3

Values of the parameters defining the contact area (ratio $=1.5$ )

\begin{tabular}{llll}
\hline$P(\mathrm{~N})$ & 10 & 15 & 20 \\
\hline$A(\mu \mathrm{m})$ exp. & 240 & 260 & 280 \\
$B(\mu \mathrm{m})$ & 60 & 80 & 100 \\
$\theta(\mathrm{mm})$ & 58 & 68 & 73 \\
\hline
\end{tabular}

Table 4

Contact area dimensions

\begin{tabular}{lllll}
\hline & $\begin{array}{l}\text { Final } \\
\text { statc }\end{array}$ & $\begin{array}{l}\text { Intermediate } \\
\text { state }\end{array}$ & $\begin{array}{l}\text { Modified Hertz's } \\
\text { pressure }\end{array}$ & $\begin{array}{l}\text { Initial Hertz's } \\
\text { pressure }\end{array}$ \\
\hline$A(\mathrm{sm})$ & 280 & 140 & 100 & 73 \\
$B(\mathrm{~mm})$ & 100 & 100 & 100 & 73 \\
$A / B$ & 2.8 & 1.4 & 1 & 1 \\
$P_{0} / k$ & 1.5 & 2.9 & 4.1 & 9
\end{tabular}

$P=20 N, \mu=0.8$

0.5. In Figs. 5-8 four quantities are presented as function of the angular position $\theta$ along the section of the sliding path in stabilized cycles with loading centred at $\theta=0$. These quantities are the tangential displacement $u_{m}$ the normal displace-
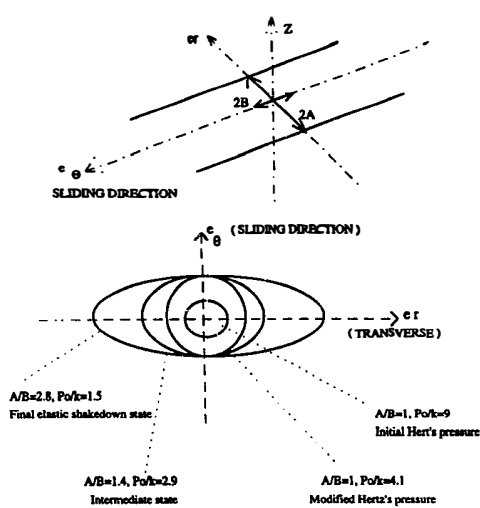

Fig. 11. Contact area related to loading.

ment $u_{z}$, the plastic shear strain $\epsilon_{q}^{p}$ and the equivalent plastic shear strain $E_{\text {equivelent }}$. This case corresponds to plastic shakedown state as can be seen in Figs. 7 and 8. 


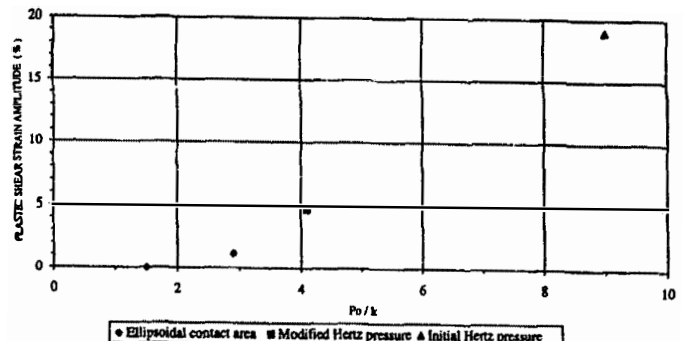



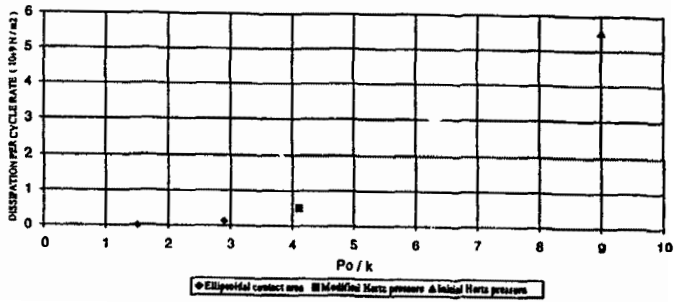

Fig. 13. Variation dissipation rate per cycle for $P=20 \mathrm{~N}$ and $\mu=0.8$.

Calculations have been performad for the three normal loadings discussed previously and for the following values of the friction coefficient: $0.2,0.5,0.6,0.7,0.8,0.9$ and 1 .

The plastic shear strain ampliude $\Delta \epsilon_{\mathbb{Q}}^{\mathrm{P}}$, which seems to be the most significant paraineter for fatigue, is presented in Fig. 9 versus the load parameter $P_{\mathrm{o}} / k$ and the various values of the friction parameters are considered. The dissipation per cycle near the surface given by:

$$
\int_{0}^{T}\left(\sigma-c e^{p}\right): e^{p} d t
$$

for kinematic hardening material, is represented in Fig. 10. These two quantities, the plastic shear strain amplitude and the dissipation rate, are the most relevant for wear modelling in relation to fatigue properties.

To account for the evolution of the contact area during a wear test, it is necessary to perform other computations with varying contact area and pressure tistribution. As suggested by Kapoor and Johnson [13], the wear phenomena will induce variations of conformity and consequently the area of contact and the pressure distribution. Following these authors the stabilized shape corresponds to the elastic shakedown state. They also give the shakedown loads with the aspect ratio $B / A$ ( $A$, and $B$ are the radii of the contact ellipse) and for different values of the friction coefficient $\mu$. For $\mu=0.8$, the corresponding shakedown values $P_{0} / k$ is approximately equal to 1.5 .

To estimate the contact parameters which will be used we proceed as follows. We measure the width of the sliding track $A$ and assume that $A$ corresponds to one of the transverse lengths of the contact. Taking $P_{\mathrm{o}} / k=1.5$, we compute the

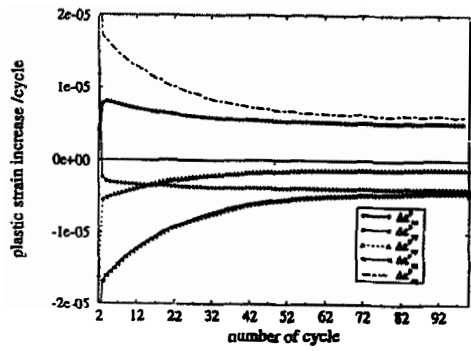

Fig. 14. Evolution per cycle of the ratchetting mate $P_{0} / k=3.5, \mu=0.5$. 
other dimension using the classical Hertz equation: $P=$ $(2 / 3) \times P_{\mathrm{o}} \pi A B$ with $P_{\mathrm{o}}=1.5 \mathrm{k}$. Theresultsobtained aresummarized in Table 3 where $a$ represents the initial contact radius.

Note that the contact dimension in the sliding direction remains rather constant. It is for this reason that we suppose $B$ to be nearly constant from the beginning to the end of the wear experiment. In the following calculations, only the transverse dimension of the contact area $A$ changes.

For instance, for $P=20 \mathrm{~N}$ and $\boldsymbol{\mu}=0.8$ the following cases were studied and presented in Fig. 11 and summarized in Table 4.

The variation in resulting shear strain amplitude $\Delta \epsilon_{\theta z}$ is presented in Fig. 12. The results obtained with the modified Hertz's distribution, which are much lower than the initial one, seem to be more realistic because the evolution of the geometry of the contact was accounted for.

Fig. 13 presents the dissipation rate under the loading.

Finally, another important quantity, the ratchetting rate, can also be estimated by our computation method. The ratchetting rate depends explicitly on the constitutive equation [5]. Using the same non-linear kinematic hardening law they considered, we derive numerically the ratchetting rate. To avoid lengthy computations, only $2 \mathrm{D}$ cases were examined for $P_{\mathrm{o}} / \boldsymbol{k}$. For instance, the evolution of the ratchetting rate for plastic strain components is given in Fig. 14 for $\boldsymbol{\mu}=\mathbf{0 . 5}$.

The ratchetting rate decreases rapidly and tends toward a stabilized value of $10^{-5}$ per cycle, whicil confirms the order of magnitude given in Ref. [4].

\section{Conclusions}

The first difficulties to overcome for modelling wear phenomena are to evaluate the physical quantities which are responsible for the degradation of material. Until now, only approximate computational methods for particular constitutive equations, e.g. perfect plasticity, are available. We try to develop systematic and reliable computational tools to respond to this deficiency. These metivids should be applicable to general elasto-plastic constitutive equations including non-linear kinematic hardening and isotropic hardening, improving our material properties description. This is achieved by a steady-state elasto-plasticity algorithm. In this work, we try to apply this method to wear problems in simulating a pin-on-disk experiment. The flexibility of the method for evaluating the mechanical quantities near the contact region is demonstrated.

This constitutes a first step in interpreting wear phenomena in relation to other material properties such as fatigue or ductility.

\section{References}

[1] H.C. Meng, and K.C. Ludema, Wear mosels and predictive equations: Tixisi fuili ano convent, Wear, 181-183 (1995) 443-457.

[2] K. Kim and K.C. Ludema, A correlation between low cycle favigue properties and scuffing properties of 4340 steel, J. Tribol. II7 (1995) 617-621.

[3] K.L. Juhnson. Contact mechanics and the wear of metals, Wear, 190 (1995) 162-170.

[4] J.M. Challen and P.L.B. Oxley, An explanation of different regimes of friction and wear using asperity deformation models, Wear, 53 (1979) 229-243.

[5] A.F. Bower. and K.L. Johnson, The influence of stain handening on cumulative plastic deformation in rolling and sliding line contact, $J$. Mech. Phys. Solids, 37 (4) (1989) 471-493.

[6] V. Barghava and G.T. Hahn and C.A. Rubin, A.S.M.E. J. Appl. Mech. 52 (1985) 67-82.

[7] k. Dang Van and H. Maitournara, Steady-state flow in classical elastoplasticity: Applications to repened rolling and sliding contaa. J. Mech. Phys. Solids, 41 (11) (1993) 1691-1710.

[8] K. Dang Van and H. Maitoumam, Thermomechanical state near rolling consect area, in D. Dowson a al. (eds.), Dissipative Process in Tribology, Elsevier, Amsterthr, 1994.

[9] K. Dang Van and H. Maitouman, Elasto-plastic Calculations of the Mechanical State in Reciprocating Moving Contocts: Application to Fretting Fatigue, Mechanical Engineering Publications, Loodon, 1994, pp. 161-168.

[10] E. Hanus, H. Maitournam and K. Dang Van, Pressure rolling: modelling by a steady stexe flow analysis and comperison with experimental data, Int. J. Solids Struct., Vol. 33 (25) (1996) pp.3753.

[11] C. Papaphilippou, Enude tribologique d'une fonte à graphive speroìdal avant et appits trempe L.A.S.E.R., application au tritement L.A.S.E.R. par points, These, Ecole Necionale des Mines de Paris, 1994.

[12] K.L. Johnson. The application of stakedowo principles in rolling and sliding contact, J. Mech. A. Solids, 11 (1992) 155-172.

[13] A. Kapoor and K.L. Johnson, Effect of changes in contact geometry on shakedown of surfaces in rolling/sliding contact. J. Mech. Phys. Solids, 34 (3) (1992) 223-239. 